\title{
Comparative Study of Serum Leptin and Insulin Resistance Levels Between Korean Postmenopausal Vegetarian and Non-vegetarian Women
}

\author{
Mi-Hyun Kim, Yun-Jung Bae ${ }^{2 *}$ \\ 'Department of Food and Nutrition, Korea National University of Transportation, Jeungpyeong 368-701, Korea \\ ${ }^{2}$ Division of Food Science and Culinary Arts, Shinhan University, Uijeongbu 480-701, Korea
}

The present study was conducted to compare serum leptin and insulin resistance levels between Korean postmenopausal longterm semi-vegetarians and non-vegetarians. Subjects of this study belonged to either a group of postmenopausal vegetarian women $(n=54)$, who maintained a semi-vegetarian diet for over 20 years or a group of non-vegetarian controls. Anthropometric characteristics, serum leptin, serum glucose, serum insulin, insulin resistance (HOMA-IR; Homeostasis Model Assessment of Insulin Resistance), and nutrient intake were compared between the two groups. The vegetarians showed significantly lower body weight $(p<0.01)$, body mass index $(p<0.001)$, percentage $(\%)$ of body fat $(p<0.001)$, and serum levels of leptin $(p<$ $0.05)$, glucose $(p<0.001)$, and insulin $(p<0.01)$, than the non-vegetarians. The HOMA-IR of the vegetarians was significantly lower than that of the non-vegetarians $(p<0.01)$ after adjustment for the $\%$ of body fat. A long-term vegetarian diet might be related to lower insulin resistance independent of the \% of body fat in postmenopausal women.

Key Words: Vegetarians, Non-vegetarians, Insulin resistance, Leptin

${ }^{*}$ Corresponding author Yun-Jung Bae

Address Division of Food Science and Culinary Arts, Shinhan University, 95 Hoam-ro, Uijeongbu 480-701, Korea

Tel +82-31-870-3572 Fax +82-31-870-3519

E-mail byj@shinhan.ac.kr

Received June 22, 2015

Revised July 7, 2015

Accepted July 9, 2015

\section{Introduction}

Population aging resulting from the increase of elderly population is a phenomenon observed in many countries around the world, including Korea, where elderly population is steadily increasing [1]. The average life span of Korean is currently reported as 78.5 years for men and 86.1 years for women, resulting in a 7-year greater life expectancy for women, and this led to an increased interest in the lifestyle and health in middle- and older-aged women [1]. The recent interest in health tends to focus on a change in lifestyle or diet. As the meat-based diet has have been suspected of increasing the incidence of chronic diseases, there has been an increase in the public interest in vegetarian diets as well as in campaigns to encourage eating more vegetables [2].

Vegetarian diets are predominantly plant-based. Compared to people who eat meat, vegetarians typically eat more mixed
This is an Open Access article distributed under the terms of the Creative Commons Attribution Non-Commercial License (http://creativecommons.org/licenses/by-nc/3.0/) which permits unrestricted non-commercial use, distribution, and reproduction in any medium, provided the original work is properly cited. 


\section{Kim MH et al.}

grains, peas and soy products, fruit, and green and yellow vegetables, and intake less sodium, saturated fatty acids, and dietary cholesterol, which decreases their risk of cardiovascular disease [3,4]. The traditional Korean diet consists primarily of grain and vegetables. In numerous studies, Western countries have been clearly shown to have a long history of health problems caused by excessive intake of animal-based foods such as meat [5-7].

Recently, some studies have focused on the effect of vegetarian diets on insulin resistance $(\mathrm{IR})[6,8]$. IR is a condition associated with a decrease in the effects of insulin, which has been considered to be the cause of type 2 diabetes and recently has been shown to be a symptom of high blood pressure and cardiovascular diseases, including metabolic syndrome $[9,10]$. In a case-controlled study in Japan that included 513 subjects, the dietary factors related to IR were analyzed, and a high fat/Western dietary pattern was found to have a significantly positive correlation with IR [11]. A study in Taiwan that included 391 vegetarian women and 315 non-vegetarian women reported that even after adjusting for the confounding factors, vegetarians had a lower risk of Homeostasis Model Assessment of Insulin Resistance (HOMA-IR) than nonvegetarians [6].

Still only a limited number of studies has been reported the aspect of vegetarian diet in metabolic disorders such as IR. The objective of this study was to compare and analyze the dietary intake of vegetarian and non-vegetarian diet groups of postmenopausal women and the serum indices related to IR.

\section{Materials and Methods}

\section{Subjects}

The study involved 102 postmenopausal women in age of 47 to 85 years old in Seoul and Gyeonggi-do. Vegetarian women $(n=54)$ and non-vegetarian women $(n=48)$ were recruited for the study through the official advertisements. All of the vegetarians had been on a semi-vegetarian diet that restricted the intake of meat and some food groups for at least 20 years. Composition of subjects' age in each group was matched by 5 year term. The inclusion criteria were that subjects were in apparently good health, were nonsmokers, consumed alcoholic drinks in moderation, and did not take any nutritional supplements. This study was conducted according to the guidelines laid down in the Declaration of Helsinki. Written informed consent was obtained from all subjects.

\section{Anthropometric assessments}

Height and weight were measured by an automatic measuring instrument (DS-102, JENIX, Seoul, Korea), with the subjects wearing comfortable light clothes and without shoes. Using the height and weight, we calculated the body mass index (BMl: body mass index $=$ Weight $\left.(\mathrm{kg}) /[\text { Height }(\mathrm{m})]^{2}\right)$, and we measured the body fat (\%) with a body fat analyzer (TBF-105, TANITA, Tokyo, Japan).

\section{Dietary intake survey}

Dietary intake survey was performed under the guidance of the investigator using a $24-h$ recall method for non-consecutive 3 days by well-trained interviewers. Food models, dishes, glasses, and spoons of various sizes were used to improve the accuracy of portion-size estimates. The nutrition and food intake by food groups was analyzed using a nutritional analysis program, Can-Pro 2.0 (The Korean Nutrition Society), based on the results of the survey.

Collection of blood and analysis of the biochemical indices Blood samples were drawn in the morning hours, after a 12-h overnight fast. The samples were left at room temperature for $30 \mathrm{~min}$, centrifuged for $15 \mathrm{~min}$ at 2,500 rpm to separate the serum, and then stored at $-70{ }^{\circ} \mathrm{C}$. The serum cholesterol, HDL cholesterol, triglyceride (TG), and plasma glucose were analyzed with a commercial kit (Boehringer Mannheim GmbH, Mannheim, Germany) and an automatic chemistry analyzer (HITACHI 747, Tokyo, Japan), whereas the serum LDL cholesterol concentration was calculated according to Friedewald's formula [12]. Serum leptin was determined using a human leptin RIA kit (Linco Research, St. Charles, MO, USA), and serum insulin was measured by enzyme immunoassay (Immulite 2000, SIEMENS, IL, USA). To determine IR, we used the HOMA-IR index. Fasting plasma glucose levels and the insulin concentration were used to calculate the HOMA-IR, according to the formula of Matthews et al [13]: HOMA-IR = [fasting plasma glucose $(\mathrm{mmol} / \mathrm{L}) \times$ fasting plasma insulin $(\mu / \mathrm{U} /$ $\mathrm{mL}$ )]/22.5. Additionally, referring to other studies, a value of HOMA-IR above 2.0 was classified as IR.

\section{Statistical analysis}

All analyses were performed using SAS ${ }^{\mathrm{TM}}$ (version 9.2, SAS Inc., Cary, NC, USA). The data were expressed as means \pm standard deviations (SD). The significance of differences between the vegetarian diet group and non-vegetarian diet group for continuous variables such as the anthropometric in- 
dex, the intake amount by food group and the nutrient intake amounts, blood lipid and leptin levels, insulin, glucose, and IR were analyzed by student's t-test. Blood indices such as serum leptin, insulin, and fasting plasma glucose levels and IR are influenced by body fat. To correct for this influence, further analysis was performed by adjusting for percent body fat in the ANCOVA (analysis of covariance). The significance level of all data was $p<0.05$.

\section{Results}

\section{General characteristics}

The age and anthropometric measurements of the subjects are presented in Table 1. The average age of the vegetarians and the non-vegetarians was 62.6 and 60.2 years, respectively, with no significant difference. The height was not significantly different among the vegetarians and non-vegetarians, whereas the average weight in the non-vegetarian group was 58.8 $\mathrm{kg}$, which was significantly higher than that in the vegetarian group, $53.9 \mathrm{~kg}(\mathrm{p}<0.01)$. The BMl and body fat percentage of the non-vegetarians were $25.2 \mathrm{~kg} / \mathrm{m}^{2}$ and $37.4 \%$, respectively, which were significantly higher than those in the vegetarian diet group, $22.6 \mathrm{~kg} / \mathrm{m}^{2}$ and 30.9\%, respectively (each $p<0.001$ ).

\section{Dietary intake}

The total food intake amount and the intake by food group are presented in Table 2 . The average daily total food intake of the vegetarians and non-vegetarians was $969.4 \mathrm{~g}$. and $873.8 \mathrm{~g}$, respectively, without a significant difference. Compared with the non-vegetarians, the vegetarian diet group had a higher intake of potatoes and starches ( $p<0.01)$, soybean and soy products ( $p<0.05)$, nuts and seeds ( $p<0.01)$, vegetables ( $p$ $<0.001)$, and seaweeds ( $<<0.05)$, and a significantly lower intake of meats $(p<0.001)$, milks ( $p<0.001)$, beverages ( $p<$ 0.05), and seasonings ( $p<0.001)$.
The energy and nutrient intake is presented in Table 3. The total daily energy intake was $1405.9 \mathrm{kcal}$ in the vegetarians and $1365.9 \mathrm{kcal}$ in the non-vegetarians; the difference between two groups was not significant. The vegetarians consumed a significantly higher amount of plant protein $(p<0.001)$ and carbohydrates $(p<0.05)$ than the non-vegetarians.

\section{Serum lipids, leptin, insulin, glucose, and HOMA-IR}

The serum lipid levels of the subjects are shown in Figure 1. The serum concentration of triglyceride and HDL-cholesterol was not significantly different between the two groups. The serum total cholesterol and LDL-cholesterol levels were 184.4 $\mathrm{mg} / \mathrm{dL}$ and $111.2 \mathrm{mg} / \mathrm{dL}$, respectively, for the vegetarians and $209.5 \mathrm{mg} / \mathrm{dl}$ and $133.5 \mathrm{mg} / \mathrm{dL}$, respectively, for the non-vegetarians $(p<0.001, p<0.01)$.

The serum leptin, insulin, and fasting blood glucose levels and HOMA-IR for the subjects are shown in Figure 2. The serum levels of leptin ( $p<0.05)$, insulin ( $p<0.01)$, and fasting blood glucose $(p<0.001)$ in the non-vegetarians were significantly higher than the levels in the vegetarians; the HOMAIR for the non-vegetarians was 4.6, which was significantly higher than the HOMA-IR of 2.9 for the vegetarians $(p<0.001)$. The ratio of $I R$ in each group (HOMA-IR > 2.0) was analyzed (data not shown), and $68.5 \%$ of the vegetarian diet group and $95.8 \%$ of the non-vegetarian diet group showed IR ( $p<$ 0.001). The serum levels of leptin, insulin, and blood glucose are reported to be closely related to body fat, and the results for each index, after adjusting for the body fat, are shown in Figure 3. After adjusting for the percentage of body fat, the significant difference disappeared in the serum leptin and insulin levels between the non-vegetarians and the vegetarians. The vegetarian diet group showed significantly lower blood glucose and HOMA-IR than the non-vegetarian diet group ( $p$ $<0.01$ ), even with the exclusion of the influence of body fat.

Table 1. Anthropometric measurements of the subjects

\begin{tabular}{lccc}
\hline & $\begin{array}{c}\text { Vegetarians } \\
(\mathbf{n}=\mathbf{5 4})\end{array}$ & $\begin{array}{c}\text { Non-vegetarians } \\
(\mathbf{n}=\mathbf{4 8})\end{array}$ & Significance $^{+}$ \\
\hline Age, year & $62.6 \pm 9.0^{*}$ & $60.2 \pm 5.7$ & $\mathrm{NS}^{+}$ \\
Height, $\mathrm{cm}$ & $154.1 \pm 5.8$ & $152.6 \pm 5.0$ & $\mathrm{NS}$ \\
Weight, $\mathrm{kg}$ & $53.9 \pm 9.0$ & $58.8 \pm 6.8$ & $\mathrm{p}<0.01$ \\
Body mass index, $\mathrm{kg} / \mathrm{m}^{2}$ & $22.6 \pm 3.4$ & $25.2 \pm 3.1$ & $\mathrm{p}<0.001$ \\
\% body fat & $30.9 \pm 7.6$ & $37.4 \pm 6.9$ & $\mathrm{p}<0.001$ \\
\hline
\end{tabular}

*Mean \pm standard deviation; ${ }^{+}$Significance as determined by Student's t-test; ${ }^{*}$ Not significant. 


\section{Kim MH et al.}

Table 2. Amount of subjects' food intakes from each food group

\begin{tabular}{|c|c|c|c|}
\hline & $\begin{array}{l}\text { Vegetarians } \\
(n=54)\end{array}$ & $\begin{array}{l}\text { Non-vegetarians } \\
\quad(n=48)\end{array}$ & Significance $^{+}$ \\
\hline Total food, g/day & $969.4 \pm 245.1^{*}$ & $873.8 \pm 316.6$ & $\mathrm{NS}^{*}$ \\
\hline Cereals, g/day & $284.2 \pm 109.6$ & $256.0 \pm 72.5$ & NS \\
\hline Potato and starches, g/day & $55.8 \pm 76.9$ & $24.4 \pm 38.9$ & $\mathrm{p}<0.01$ \\
\hline Sugars and sweeteners, g/day & $5.1 \pm 10.4$ & $3.1 \pm 4.2$ & N.S \\
\hline Soybean and its products, g/day & $78.7 \pm 76.8$ & $49.8 \pm 39.0$ & $p<0.05$ \\
\hline Nuts and seeds, g/day & $8.4 \pm 15.6$ & $1.1 \pm 3.1$ & $p<0.01$ \\
\hline Vegetables, g/day & $282.8 \pm 115.1$ & $204.1 \pm 96.2$ & $p<0.001$ \\
\hline Fungi and mushrooms, g/day & $1.8 \pm 5.0$ & $0.6 \pm 3.4$ & NS \\
\hline Fruits, g/day & $156.5 \pm 115.1$ & $116.7 \pm 137.9$ & NS \\
\hline Meats, g/day & $1.9 \pm 4.4$ & $38.2 \pm 33.5$ & $p<0.001$ \\
\hline Eggs, g/day & $11.0 \pm 19.7$ & $10.5 \pm 15.8$ & NS \\
\hline Fish and shellfishes, g/day & $23.0 \pm 30.2$ & $30.7 \pm 30.7$ & NS \\
\hline Seaweeds, g/day & $28.8 \pm 17.8$ & $21.1 \pm 12.0$ & $\mathrm{p}<0.05$ \\
\hline Milks, g/day & $11.6 \pm 33.3$ & $74.8 \pm 104.1$ & $p<0.001$ \\
\hline Oils and fat, g/day & $4.8 \pm 3.0$ & $5.2 \pm 4.0$ & NS \\
\hline Beverages, g/day & $4.4 \pm 20.1$ & $17.9 \pm 42.1$ & $p<0.05$ \\
\hline Seasoning, g/day & $6.9 \pm 12.0$ & $9.0 \pm 20.1$ & $p<0.001$ \\
\hline
\end{tabular}

${ }^{*}$ Mean \pm standard deviation; ${ }^{\dagger}$ Significance as determined by Student's t-test; ${ }^{*}$ Not significant.

Table 3. The daily nutrient intakes of the subjects

\begin{tabular}{lccc}
\hline & $\begin{array}{c}\text { Vegetarians } \\
(\mathbf{n}=\mathbf{5 4})\end{array}$ & $\begin{array}{c}\text { Non-vegetarians } \\
(\mathbf{n}=\mathbf{4 8})\end{array}$ & Significance $^{+}$ \\
\hline Energy, kcal & $1405.9 \pm 323.4^{*}$ & $1365.9 \pm 410.5$ & NS $^{\ddagger}$ \\
Protein, g & $50.0 \pm 15.4$ & $52.4 \pm 18.3$ & NS \\
Animal protein & $8.5 \pm 6.9$ & $19.8 \pm 12.6$ & $\mathrm{p} 0.001$ \\
Plant protein & $41.5 \pm 14.0$ & $32.6 \pm 9.9$ & $\mathrm{p}<0.001$ \\
Fat, g & $23.4 \pm 9.8$ & $27.5 \pm 14.3$ & $\mathrm{NS}$ \\
\hline Animal fat & $2.9 \pm 2.7$ & $10.2 \pm 7.0$ & $\mathrm{p}<0.001$ \\
\hline Plant fat & $20.4 \pm 9.5$ & $17.3 \pm 12.2$ & $\mathrm{NS}$ \\
Carbohydrate, g & $251.0 \pm 57.7$ & $227.5 \pm 61.8$ & $\mathrm{p}<0.05$ \\
\hline Carbohydrate, in kcal, \% & $71.7 \pm 5.4$ & $67.6 \pm 6.2$ & $\mathrm{p}<0.001$ \\
Protein, in kcal, \% & $14.2 \pm 2.6$ & $15.2 \pm 2.5$ & $\mathrm{NS}$ \\
\hline Fat, in kcal, \% & $14.8 \pm 4.5$ & $17.4 \pm 5.1$ & $\mathrm{p}<0.01$ \\
\hline
\end{tabular}

${ }^{*}$ Mean \pm standard deviation; ${ }^{\dagger}$ Significance as determined by Student's t-test; ${ }^{*}$ Not significant.

\section{Discussion}

The beneficial effects of a vegetarian diet in improving IR and glucose metabolism are well documented. Hung et al. [14] reported that vegetarians have notably lower fasting plasma glucose and serum insulin levels but higher insulin sensitivity than the genenral diet group. Having vegetarian diet is associated with reducing insulin secretion but the increase of glucagon secretion $[15,16]$. In a study in Taiwan, Chiang et al. [6] 


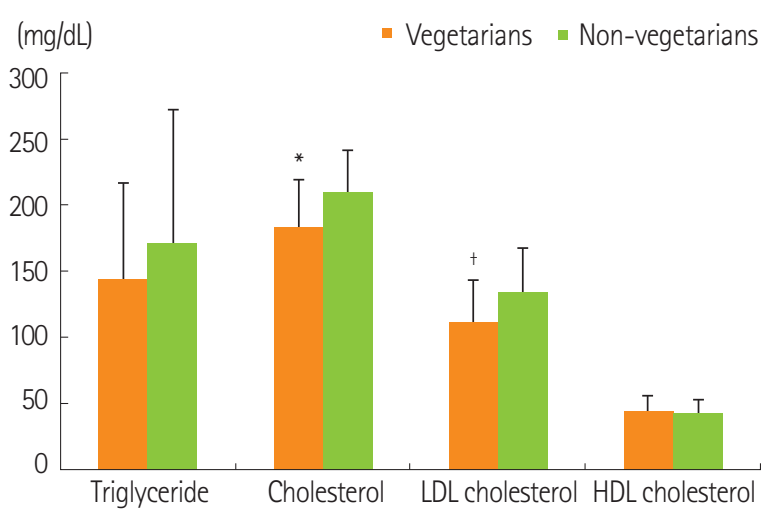

Figure 1. Blood lipid parameters of the subjects. Significance as determined by Student's t-test. ${ }^{*} p<0.001 ;{ }^{\dagger} p<0.01$.

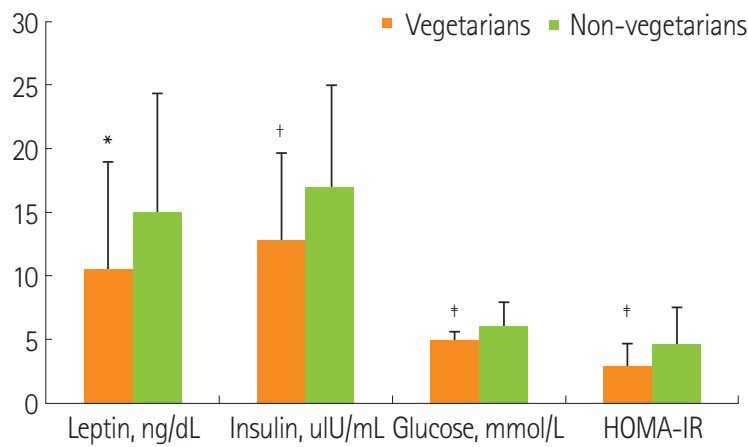

Figure 2. The levels of serum leptin, insulin, glucose and HOMA-IR of the subjects. Significance as determined by Student's t-test. ${ }^{*} p<0.05$ $i^{\dagger} p<0.01 ;{ }^{\ddagger} p<0.001$.

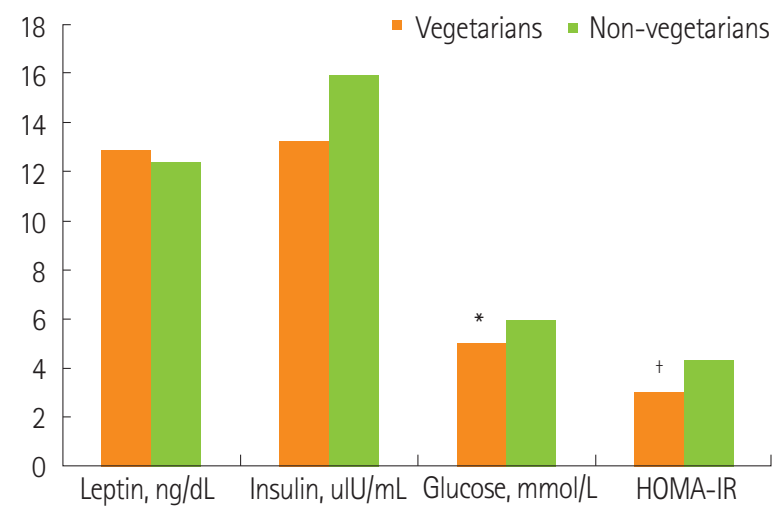

Figure 3. The levels (estimated mean) of serum leptin, insulin, glucose and HOMA-IR of the subjects adjusting for $\%$ body fat. Significance as determined by ANCOVA test. ${ }^{*} p<0.01 ;{ }^{\dagger} p<0.05$. reported a significant relationship between a vegetarian diet and IR, after adjusting for confounding factors including age, menopausal status, and physical activity. In addition, for the lacto-ovo-, pesco-, and other semi-vegetarian subjects, the risk of type 2 diabetes was significantly lower than the risk in the non-vegetarian diet group, even after adjusting for several confounding factors [17]. In this study, the serum levels of leptin, insulin, fasting blood glucose, and HOMA-IR level were significantly lower in the Korean vegetarians who maintained a vegetarian diet for a long period of time than the Korean non-vegetarians. These results were comparable to those of previous reports [6,17-19].

In many studies including this study, the weight and BMI of vegetarians were significantly lower than those of nonvegetarians $[6,20,21]$. It is interpreted that the vegetarian diet increases the intake of dietary fiber and lowers the intake of saturated fatty acids, which could reduce the amount of body fat of subjects in this study [22]. It is reported to have protective effects against overweight and obesity [23]. Some studies report that the relationship could be influenced by the lifestyle and intake of protein and dietary fiber of the subjects [24]. In our study, the BMI and body fat percentage of the vegetarians were significantly lower than those of the nonvegetarians. This physiological difference between the two groups could have an effect on the glucose metabolism and IR. Valachovicová et al. [25] compared the fasting glucose, insulin, and HOMA-IR in vegetarians and omnivores with normal weight; although there was no difference in BMI between the two groups, vegetarians' fasting glucose, insulin, and IR were significantly lower than the omnivores'. Interestingly, in our study, after adjusting for body fat percentage, the significant difference in fasting blood glucose and HOMA-IR between the groups persisted (Figure 3). However, the statistically significant difference in serum leptin and insulin between the two groups disappeared after adjusting for body fat percentage.

Vegetarians eat fruits, grain, nuts, beans, and vegetables and restrict their intake of certain food groups such as meat, fish, and poultry [26], and these are different dietary features from non-vegetarian. In this study, the difference in the energy intake between the groups was slight, although the energy intake from fat was significantly lower in the vegetarian diet group $(p<0.01)$. The intake of animal protein and fat was significantly lower in the vegetarians than in the non-vegetarians whereas the intake of plant protein and energy from carbohydrates was significantly higher in the vegetarians. The vegetarians consumed notably greater amounts of plant-based foods 


\section{Kim MH et al.}

such as beans, seeds, vegetables, and seaweeds than did the non-vegetarian diet group, whereas the intake of the animalbased food was significantly lower.

Number of people who adopt vegetarian diets is increasing in many countries [27]. It has been reported that a low fat vegetarian diet improves the control of glycemia and lipid levels $[14,28]$ and reduces the risk of cardiovascular disease [29]. The preceding studies report a significant difference in the blood lipid levels of subjects who follow a vegetarian diet compared to those of the subjects in the non-vegetarian diet group $[30,31]$. A meta-analysis of randomized controlled trials shows that a low-fat diet for women lowers total cholesterol, HDL cholesterol, and LDL cholesterol, without a meaningful relationship with TG [32]. In this study, the subjects were postmenopausal women, and the total cholesterol and LDL-cholesterol levels in the vegetarian diet group were significantly lower than those in the non-vegetarian diet group, whereas there was no meaningful difference in the TG between the groups. Also, leptin is an adipokine dysregulated in obese and insulin-resistant subjects [33]. Insulin and leptin signaling constitute the adipo-insular axis, which contributes to the regulation of nutrient and energy balance in the body. Dysregulation of the adipo-insular axis may contribute to the progression of IR $[34,35]$. In our study, the serum levels of leptin and insulin were significantly lower in Koreans who maintained a vegetarian diet than the Korean non-vegetarians.

Vegetarian or plant-based diets usually provide a plenty of nutrients and bioactive substances such as monounsaturated and polyunsaturated fatty acids, soy or vegetable proteins, fiber, antioxidant vitamins, and a variety of phytochemicals through vegetables, fruits, whole grains, cereals, nuts, seeds, legumes, and soybeans [36]. Regarding the relationship of various diseases and a vegetarian diet, studies have reported an association of a vegetarian diet with diabetes and IR $[19,36]$. Wolfram \& Ismail-Beigi [37] reported that vegetable and whole grain intake had a significant relationship with insulin sensitivity at the cellular level in 31- to 45-year-old Taiwanese women.

This study has some limitations. First, the study had a small sample size and thus needs to be repeated with a larger population. There may be a difference in IR based on the level of the vegetarian diet (lacto-ovo, pesco, semi-vegetarian) followed by the subjects; further research specifically investigating IR according to the vegetarian diet level of the subjects and involving higher numbers of subjects are necessary. Second, a detailed analysis of the nutrients or bioactive substanc- es absorbed from a vegetarian diet is lacking, which is a limitation of the study. The effect of vegetarianism on IR differs by the nutritional components of the vegetarian diet. Despite these limitations, the results of this study are strengthened by studying the subjects who have maintained a long-term (over 20 years) vegetarian diet.

\section{Conclusion}

Vegetarianism is a dietary regime based predominantly on food from plant sources; it is related to heart disease, cancer, obesity and various chronic diseases. In this study, postmenopausal women were grouped into a vegetarian diet group and a non-vegetarian diet group, and their dietary intake and serum indices related to IR were analyzed. Compared with the middle-aged women who followed a non-vegetarian diet, the weight, BMI, and body fat percentage of those who followed a vegetarian diet were significantly lower, and the results of serum leptin, insulin and fasting glucose showed same trend. Furthermore, the HOMA-IR levels of the vegetarian subjects were lower than those of the non-vegetarian women, even after adjusting for the percentage of body fat. The results of this study support the beneficial effect of a long-term semivegetarian diet specifically, that it can improve the IR and glucose metabolism in postmenopausal women.

\section{Conflict of Interest}

The authors have declared no conflict of interest.

\section{References}

1. Statistics Korea. 2013 Life tables. Dajeon: Statistics Korea; 2014.

2. Le LT, Sabaté J. Beyond meatless, the health effects of vegan diets: findings from the Adventist cohorts. Nutrients 2014;6:2131-47.

3. Ferdowsian HR, Barnard ND. Effects of plant-based diets on plasma lipids. Am J Cardiol 2009;104:947-56.

4. Flock MR, Green MH, Kris-Etherton PM. Effects of adiposity on plasma lipid response to reductions in dietary saturated fatty acids and cholesterol. Adv Nutr 2011;2:261-74.

5. Yang SY, Zhang HJ, Sun SY, Wang LY, Yan B, Liu CQ, Zhang W, Li XJ. Relationship of carotid intima-media thickness and duration of vegetarian diet in Chinese male vegetarians. Nutr Metab (Lond) 2011;8:63-9.

6. Chiang JK, Lin YL, Chen CL, Ouyang CM, Wu YT, Chi YC, Huang KC, Yang WS. Reduced risk for metabolic syndrome and insulin resistance associated with ovo-lacto-vegetarian behavior in female Buddhists: a casecontrol study. PLoS One 2013;8:e71799.

7. Agrawal S, Millett CJ, Dhillon PK, Subramanian SV, Ebrahim S. Type of vegetarian diet, obesity and diabetes in adult Indian population. Nutr J 2014;13:89-106.

8. Kahleova H, Matoulek M, Malinska H, Oliyarnik O, Kazdova L, Neskudla T, Skoch A, Hajek M, Hill M, Kahle M, Pelikanova T. Vegetarian diet 
improves insulin resistance and oxidative stress markers more than conventional diet in subjects with Type 2 diabetes. Diabet Med 2011;28:549-59.

9. Grundy SM. Hypertriglyceridemia, insulin resistance, and the metabolic syndrome. Am J Cardiol 1999;83:25F-9F.

10. Mikhail N. The metabolic syndrome: insulin resistance. Curr Hypertens Rep 2009;11:156-8.

11. Arisawa $K$, Uemura $H$, Yamaguchi $M$, Nakamoto $M$, Hiyoshi M, Sawachika F, Katsuura-Kamano S. Associations of dietary patterns with metabolic syndrome and insulin resistance: a cross-sectional study in a Japanese population. J Med Invest 2014;61:333-44.

12. Friedewald WT, Levy RI, Fredrickson DS. Estimation of the concentration of low-density lipoprotein cholesterol in plasma, without use of the preparative ultracentrifuge. Clin Chem 1972;18:499-502.

13. Matthews DR, Hosker JP, Rudenski AS, Naylor BA, Treacher DF, Turner RC. Homeostasis model assessment: insulin resistance and beta-cell function from fasting plasma glucose and insulin concentrations in man. Diabetologia 1985;28:412-9.

14. Hung CJ, Huang PC, Li YH, Lu SC, Ho LT, Chou HF. Taiwanese vegetarians have higher insulin sensitivity than omnivores. $\mathrm{Br} J$ Nutr 2006;95:129-35.

15. McCarty MF. Vegan proteins may reduce risk of cancer, obesity, and cardiovascular disease by promoting increased glucagon activity. Med Hypotheses 1999:53:459-85.

16. Kuo CS, Lai NS, Ho LT, Lin CL. Insulin sensitivity in Chinese ovo-lactovegetarians compared with omnivores. Eur I Clin Nutr 2004;58:312-6.

17. Tonstad S, Butler T, Yan R, Fraser GE. Type of vegetarian diet, body weight, and prevalence of type 2 diabetes. Diabetes Care 2009;32:7916.

18. Amini M, Esmaillzadeh A, Shafaeizadeh S, Behrooz J, Zare M. Relationship between major dietary patterns and metabolic syndrome among individuals with impaired glucose tolerance. Nutrition 2010;26:986-92.

19. Gadgil MD, Anderson CA, Kandula NR, Kanaya AM. Dietary patterns in Asian Indians in the United States: an analysis of the metabolic syndrome and atherosclerosis in South Asians Living in America study. J Acad Nutr Diet 2014;114:238-43.

20. Berkow SE, Barnard N. Vegetarian diets and weight status. Nutr Rev 2006;64:175-88.

21. Burke LE, Styn MA, Steenkiste AR, Music E, Warziski M, Choo J. A randomized clinical trial testing treatment preference and two dietary options in behavioral weight management: preliminary results of the impact of diet at 6 months--PREFER study. Obesity (Silver Spring) 2006;14:2007-17.

22. Miller WC, Niederpruem MG, Wallace JP, Lindeman AK. Dietary fat, sug- ar, and fiber predict body fat content. J Am Diet Assoc 1994;94:612-5.

23. Appleby PN, Thorogood M, Mann Jl, Key TJ. Low body mass index in non-meat eaters: the possible roles of animal fat, dietary fibre and alcohol. Int J Obes Relat Metab Disord 1998;22:454-60.

24. Spencer EA, Appleby PN, Davey GK, Key TJ. Diet and body mass index in 38000 EPIC-Oxford meat-eaters, fish-eaters, vegetarians and vegans. Int J Obes Relat Metab Disord 2003;27:728-34.

25. Valachovicová M, Krajcovicová-Kudlácková M, Blazicek P, Babinská K. No evidence of insulin resistance in normal weight vegetarians. A case control study. Eur J Nutr 2006;45:52-4.

26. Sabaté J, Ratzin-Turner R. Vegetarian nutrition. 1st ed. Boca Raton (FL): CRC Press; 2001.

27. Craig WJ. Health effects of vegan diets. Am J Clin Nutr 2009;89:1627S$33 \mathrm{~S}$.

28. Barnard ND, Cohen J, Jenkins DJ, Turner-McGrievy G, Gloede L, Jaster B, Seidl K, Green AA, Talpers S. A low-fat vegan diet improves glycemic control and cardiovascular risk factors in a randomized clinical trial in individuals with type 2 diabetes. Diabetes Care 2006;29:1777-83.

29. Dominique Ashen M. Vegetarian diets in cardiovascular prevention. Curr Treat Options Cardiovasc Med 2013;15:735-45.

30. Fu CH, Yang CC, Lin CL, Kuo TB. Effects of long-term vegetarian diets on cardiovascular autonomic functions in healthy postmenopausal women. Am J Cardiol 2006;97:380-3.

31. Huang $Y W$, Jian $Z H$, Chang $H C$, Nfor ON, Ko PC, Lung CC, Lin LY, Ho CC, Chiang YC, Liaw YP. Vegan diet and blood lipid profiles: a crosssectional study of pre and postmenopausal women. BMC Womens Health 2014;14:55-60.

32. Wu L, Ma D, Walton-Moss B, He Z. Effects of low-fat diet on serum lipids in premenopausal and postmenopausal women: a meta-analysis of randomized controlled trials. Menopause 2014;21:89-99.

33. Hotta $K$, Funahashi $T$, Bodkin NL, Ortmeyer HK, Arita Y, Hansen BC, Matsuzawa $Y$. Circulating concentrations of the adipocyte protein adiponectin are decreased in parallel with reduced insulin sensitivity during the progression to type 2 diabetes in rhesus monkeys. Diabetes 2001;50:1126-33.

34. Barr VA, Malide D, Zarnowski MJ, Taylor SI, Cushman SW. Insulin stimulates both leptin secretion and production by rat white adipose tissue. Endocrinology 1997;138:4463-72.

35. Kieffer TJ, Habener JF. The adipoinsular axis: effects of leptin on pancreatic beta-cells. Am J Physiol Endocrinol Metab 2000;278:E1-14.

36. Sabaté J. The contribution of vegetarian diets to health and disease: a paradigm shift? Am J Clin Nutr 2003;78:502S-7S.

37. Wolfram T, Ismail-Beigi F. Efficacy of high-fiber diets in the management of type 2 diabetes mellitus. Endocr Pract 2011;17:132-42. 